\title{
Robot-Assisted Partial Nephrectomy for Renal Pelvic Urothelial Carcinoma Complicated with Renal Insufficiency: A Case Report
}

\section{Shikai Chen}

Weifang Medical University https://orcid.org/0000-0001-8627-3277

\section{Ping Li}

Affiliated Hospital of Weifang Medical University

\section{Shizhang Song}

Qingdao University

Sichuan Hou ( $\sim$ hsc640128@163.com )

Qingdao Municipal Hospital https://orcid.org/0000-0002-1629-6809

\section{Case report}

Keywords: Malignant tumor of renal pelvis, Partial nephrectomy for renal pelvic, Urothelial carcinoma, Renal insufficiency, Case report

Posted Date: November 15th, 2021

DOI: https://doi.org/10.21203/rs.3.rs-1043415/v1

License: (c) (i) This work is licensed under a Creative Commons Attribution 4.0 International License. Read Full License 


\section{Abstract}

Background Primary malignant tumors of the renal pelvis are relatively few in urinary tumors, with a high degree of malignancy and a relatively poor prognosis. Most of the pathological results are urothelial carcinoma. The standard surgical operation is full-length resection of the kidney and ureter and sleeve resection of the bladder. However, for patients with renal insufficiency or solitary kidney, clinicians find it terribly difficult to make the decision on the surgical resection and protection of the renal function. A patient with renal pelvis malignant tumor complicated with renal insufficiency was treated in our hospital.

Case presentation: A 77-year-old Chinese female with a more than 5-year history of renal dysfunction,was hospitalized due to "painless gross hematuria found for 4 months". The patient had no other significant discomfort. CT discovered that the volume of both kidneys is small, and the soft tissue density shadow was exactly at the right ureteropelvic junction. Therefore we consider the diagnosis was tumor of right renal pelvis. Patient did not accept radical surgical resection and was required to preserve the kidney. So the patient underwent robot-assisted partial nephrectomy for right renal pelvic , and the operation was successful. After surgery chemotherapy drugs were instilled into the renal pelvis through a single J-tube infusion. During nearly three years of follow-up, the patient regularly reviewed ureteroscopy and MRI/CT, no significant abnormalities were seen. Postoperative creatinine control was better and no hemodialysis was performed. The curative effect is acceptable.

Conclusions Partial nephrectomy for renal pelvic is also an option for patients with malignant tumor of renal pelvis who need to preserve their kidneys.

\section{Background}

Cancer of the renal pelvis belongs to the group of upper urinary tract urothelial carcinoma (utuc), which has a high degree of malignancy and a poor prognosis, and $7 \%-10 \%$ of patients can simultaneously have bladder cancer[1].In European and American reports, the incidence of utuc accounts for only $5 \%$ $10 \%$ of urothelial cancers[2,3]. This proportion may be slightly higher in China. The gold standard surgical operation for renal pelvic malignancies remains radical ureterectomy and sleeve resection of the bladder, especially if the tumor has an invasive, high-grade tumor (biopsy or urine cytology) or the diameter of the tumor is greater than $2 \mathrm{~cm}[4]$. However, resection of the ipsilateral kidney may lead to the development of renal dysfunction in some patients, so guidelines from the EAU also suggest that, for patients with low-risk UTUC (solitary, diameter $<2 \mathrm{~cm}$, low-grade, and no infiltrative growth on imaging), consideration may be given to preserving the ipsilateral kidney, for which no significant difference in 5year postoperative tumor specific survival is

observed compared with radical surgery, moreover, the complications of radical surgery can be avoided effectively[5]. However, for malignancies originating in the middle and upper ureter and renal pelvis, radical surgery is more recommended. But resection of one side kidney is unacceptable for those patients with severe renal dysfunction or solitary kidney, so the guidelines of EAU (2020) also propose that, for 
such patients, renal sparing surgery may also be feasible[6]. However, renal sparing surgery has not been reported in patients with high-grade urothelial carcinoma. How to instill therapy after surgery is also a concern. A patient with renal pelvis malignant tumor complicated with renal insufficiency was treated in our hospital. Partial resection of the renal pelvis was adopted and followed up for nearly three years, The curative effect is good, which is reported as follows:

\section{Case Presentation}

The patient, female, 77 years old, was hospitalized due to "painless gross hematuria found for 4 months". One month ago, CT was performed in the external hospital to indicate the right renal pelvis occupation. After that, the patient came to our outpatient department to review CT (Figure 1):1. The volume of both kidneys is small, and the soft tissue density shadow was exactly at the right ureteropelvic junction. 2 . The right bladder wall is slightly thickened, please combine with the clinical 3. Gallstone. The patient has been admitted to the hospital with "right renal pelvis space-occupying". The patient had no other obvious discomfort. The patient had a history of "hypertension" for more than 5 years, took oral "nifedipine tardyreleased tablets" treatment, and the self-reported blood pressure control was acceptable.Simultaneously, "Chronic renal insufficiency" coexisted with her for more than 5 years, and the urine volume is acceptable (about 1.5-2L per day). It is treated with oral traditional Chinese medicine, and the self-reported effect is unsatisfactory. Denied a history of drug allergy. No obvious positive signs were found in the whole body examination.Improve relevant examinations before surgery: blood routine: hemoglobin $96 \mathrm{~g} / \mathrm{L}$. Urine routine: urinary occult blood $2+$, urinary protein $2+$. Liver function: leucine aminopeptidase $332.00 \mathrm{u} / \mathrm{l}$. Renal function: creatinine $408.31 \mathrm{umol} / \mathrm{L}$, urea nitrogen $13.53 \mathrm{mmol} / \mathrm{L}$, Cystatin $3.94 \mathrm{mg} / \mathrm{L}, \beta 2$ microglobulin $18.54 \mathrm{mg} / \mathrm{L}$, uric acid $358.69 \mathrm{umol} / \mathrm{L}$. Abnormal cells were found in urine exfoliative cytology. Preliminary diagnosis: 1 . Space occupying lesion of right renal pelvis 2. Bladder tumor? 3. Chronic renal insufficiency 4. 3rd stage Hypertension 5. Gallstone.

The patient had a history of renal insufficiency for many years. Because the urine volume was acceptable, the nephrology department did not give special treatment. Repeatedly communicate with patients and their families before the operation to

inform them that they need to go to the nephrology department after radical resection. The patients and their families strongly request to retain the kidney and refuse radical resection. So the patient underwent transurethral resection of bladder lesions + robot-assisted partial nephrectomy for renal pelvic (right), and the operation was successful.

Operation process: First, cystoscopy was used to remove bladder lesions. Change the patient's body posture and connect the Da Vinci robot system into the patient's abdominal cavity. Separate the surrounding tissue and expose the renal pelvis. The excised parapelvic fat was sent for the Department of Pathology. Next, place dry gauze around the renal pelvis, which is used to protect the surrounding tissue(Figure 2). The renal pelvis was punctured with a fine needle, and the urine inside the renal pelvis was suctioned out. Cut the renal pelvis longitudinally. A vegetable-shaped tumor (Figure 3) with a 
diameter of about $3 \mathrm{~cm}$ and brittle texture can be seen. It is bleeding when touching. Explore the tumor tissue, find the tumor base, cut off the renal pelvis tissue at the lesion and send it to the Department of Pathology, Pathological report: urothelial carcinoma, no tumor tissue was found at the cutting edge. Trim the renal pelvis and ureteral wall, leave a $6 \mathrm{~F}$ double $\mathrm{J}$ tube in ureter, and re-anastomose the junction of renal pelvis and ureter. One $8 \mathrm{~F}$ single $\mathrm{J}$ tube shall be inserted into the anastomosis to ensure that the lateral hole is located in the renal pelvis and its end is fixed in the abdominal wall(figure 4). The abdominal cavity was again rinsed 3 times using sterile distilled water at $43^{\circ} \mathrm{C}$. One abdominal drainage tube was indwelling, and then the surgical wound was sutured. Final pathology: 1. (renal pelvis) high grade invasive papillary urothelial carcinoma, no nerve invasion or vascular invasion. Immunohistochemistry: CK (local +), Ki67 (50\% +), p53 (missense mutation, 75\% +), p63 +, GATA3 (+), CD138 (+), CK (+), VIM (-), CK7 (+), HER2 $(2+)$, mismatch repair gene protein dmmr immunohistochemistry: MLH1 (+), PMS2 (+), MSH2 (+), MSH6 (+), no expression loss, no microsatellite instability. 2.(renal pelvis fat) chronic inflammation of adipose fiber connective tissue, no cancer involved. 3. (bladder) chronic inflammation of mucosal tissue, formation of inflammatory granulation tissue in stroma, slightly large and deeply stained nuclei of focal mucosal epithelium, carbonized and dry tissue, and unclear morphology. On the first day after surgery, the patient was then given gemcitabine, which was slowly instilled into the renal pelvis via a single $\mathrm{J}$ tube. Renal pelvic perfusion was continuously administered from the first postoperative week, once a week for a total of eight times, and no significant adverse effects occurred in patients during the perfusion period. After leaving the hospital, the patient regularly reviewed ureteroscopy and MR/CT, no significant abnormalities were seen. Postoperative creatinine control was better and no hemodialysis was performed.

\section{Discussion}

Primary malignant tumors of the renal pelvis are relatively few in urinary tumors, with a high degree of malignancy and a relatively poor prognosis. Most of the pathological results are urothelial carcinoma. The standard surgical operation is full-length resection of the kidney and ureter and sleeve resection of the bladder. For tumors confined within the renal pelvis, ureteroscopic surgery is the favored option if preservation of the kidney is desired, percutaneous nephroscopy may be considered if necessary, or a combination of both. Percutaneous nephroscopy is more suitable for patients with urinary diversion, but the risk is that tumor tissue may seed metastases along the puncture tract[5]. Multiple studies have found no significant difference in overall survival or tumor-specific survival between percutaneous nephroscopy combined with ureteroscopy and radical surgery, but the former had a significantly higher rate of local recurrence, especially for high-grade tumors $[5,7]$. Endoscopic procedures have little trauma and can be treated many times through the natural lumen, but they carry a higher risk of bleeding, which may lead to missed lesions and the risk of transopen surgery for renal pelvic perforation[8].

The tumor diameter of this patient is greater than $2 \mathrm{~cm}$, which is a high-risk UTUC, and radical surgery should be performed[9].However, considering that the renal function of the patient is further aggravated after a radical operation, The patient comes from rural areas, and long-term hemofiltration will seriously reduce the quality of life.Moreover, some studies have found that dialysis time is related to the increased 
risk of death. For each additional year of dialysis treatment, the corresponding risk of death increases by about $6 \%[10]$.After careful consideration and considering the opinions of the patient and her family, we decided to perform a partial nephrectomy for the renal pelvic. The patients were closely followed up for nearly three years. During the follow-up, special attention was paid to whether the tumor recurred in the ipsilateral upper urinary tract[11]. So far, we have not seen tumor recurrence and metastasis. The patient's renal function is relatively stable and has not received hemofiltration or other treatments. Therefore, we think that in special cases, partial nephrectomy for renal pelvic is also an option for patients who need to preserve their kidneys. Its advantage is to retain the renal function of patients to the greatest extent, and it is easier for patients to be accepted. However, it is only applicable to patients whose tumors are limited to the renal pelvis.

Combined with past experience, we think the following measures can be taken: 1.The intraoperative pneumoperitoneum pressure was controlled and maintained at a low state. Continuous perfusion of gas to the abdominal cavity during surgery aims to maintain the surgical operating space, thus creating a gas circulation in a non resting state. During the process, suspended tumor cells are inevitably generated. The factors such as the pressure gradient inside and outside the abdomen, the low temperature of the perfusion gas and the static electricity cause the gas to leak from the incision point of the puncture point and form turbulence effect locally, so that the suspension tumor cells can be planted easily[12]. Therefore, the pressure of pneumoperitoneum should be maintained below $12 \mathrm{mmHg}$ and the flow of pneumoperitoneum less than $5 \mathrm{~L} /$ min during the operation[13]. After the operation, we should first close the pneumoperitoneum and then pull out each sleeve to avoid the tumor planting and metastasis of the incision at the puncture point caused by the "Chimney Phenomenon"[14, 15]. 2. Before incision of the renal pelvis, gauze or incision protective membrane was placed around the surgical field, and the urine inside the renal pelvis was aspirated, to prevent contamination of the surrounding tissue by urine spillage after incision and to reduce the chance of tumor implantation surrounding tissue[16]. 3 . When freeing the renal pelvis during surgery, do it as gentle as possible and avoid crushing the affected side. 4 . After the tumor tissue or its surrounding tissue is removed, it should be immediately put into specimen bag and taken out to avoid contact with other tissues or organs in the abdominal cavity. 5. After suturing the renal pelvis, the wounds were washed with $43{ }^{\circ} \mathrm{C}$ sterile distilled water for 3 times, so that some of the tumor cells were broken or dissolved and washed out with the flushing fluid[17]. 6. The tumor free principle was strictly followed during surgery, and 2 sets of surgical instruments were prepared when necessary. To avoid iatrogenic spread of tumors caused by human factors. 7 . When chemotherapy drugs are instilled into the renal pelvis through a single J-tube infusion, the rate of instillation should not be too fast because of the special characteristics of upper urinary tract dissection, which cannot be performed like the bladder by withholding urinary to storage drugs[18].

In conclusion, we consider that partial nephrectomy for renal pelvic is also an optional and effective operation under specific circumstances. However, this patient is only a case, so the safety of this operation needs to be further supported by large sample data. The patient had no metastasis and recurrence in the follow-up of the recent three years, which also benefited from the rich clinical experience of the operator, the fine operation under the 3D vision of the Da Vinci robot, and the strict follow-up after 
discharge.But this operation was not routine for malignant tumors of the renal pelvis.Only in specific situations, physicians should fully weigh the benefits and harms and consider the individual patient's circumstances.

\section{Abbreviations}

CT

computed tomography

MRI

Magnetic Resonance Imaging

\section{Declarations}

\section{Acknowledgements}

The authors thank Department of Pathology, Qingdao Municipal Hospital for performing an analysis of our patient's specimens of renal pelvis.

\section{Competing Interests}

No potential conflict of interest was reported by the author(s).

\section{Consent for Publication}

Written informed consent was obtained from the patient's family for the publication of this case report and any accompanying images.

\section{Author Contributions}

Shikai Chen: Data curation, Methodology, Writing - original draft

Ping Li: Data curation, Supervision, Writing - original draft

Shizhang Song: Conceptualization, Data curation, Formal analysis, Methodology

Sichuan Hou: Conceptualization, Methodology, Supervision, Writing - review \& editing

\section{Fundings}

This research did not receive any specific grants from funding agencies in the public, commercial, or notfor-profit sectors.

\section{Availability of Data and Materials}


The datasets used and analyzed in this report are available from the corresponding author on reasonable request.

\section{Ethics Approval and Consent Participate}

Not applicable.

\section{Author Details}

1.Weifang Medical University, Weicheng District, 7166 Baotong West Street, Weifang261053, Shandong, China

2.Affiliated Hospital of Weifang Medical University, Kuiwen District, 2428 Yuhe Street, Weifang261031, Shandong,China

3.Qingdao University, Shinan District, 308 Ningxia Street, Qingdao266071, Shandong, China

4.Department of Urology, Qingdao Municipal Hospital, Shinan District, 5 Donghai Middle Street, Qingdao266071, Shandong, China

\section{References}

1. D Fang, Zhang L, Li X, et al. Risk factors and treatment outcomes of new contralateral upper urinary urothelial carcinoma after nephroureterectomy: the experiences of a large Chinese center. J. Cancer Res. Clin. Oncol. 2014, 140(3):477-85.

2. M Rouprêt, Babjuk M, Burger $M$, et al. European Association of Urology Guidelines on Upper Urinary Tract Urothelial Carcinoma: 2020 Update. Eur. Urol.2020.

3. Munoz J J, Ellison L M. UPPER TRACT UROTHELIAL NEOPLASMS: INCIDENCE AND SURVIVAL DURING THE LAST 2 DECADES[J]. J. Urol. 2000, 164(5):1523-1525.

4. $\mathrm{G}$ Tang, $\mathrm{H} \mathrm{Hu}$, Zh Du, et al. Risk factors of renal function after radical nephroureterectomy for upper tract urothelial carcinoma. Chin.Urol.J. 2017, 38(009):692-697.

5. Seisen T, Peyronnet B, Dominguez-Escrig J L, et al. Oncologic Outcomes of Kidney-sparing Surgery Versus Radical Nephroureterectomy for Upper Tract Urothelial Carcinoma: A Systematic Review by the EAU Non-muscle Invasive Bladder Cancer Guidelines Panel. Eur. Urol.2016:1052-1068.

6. M Rouprêt, Babjuk M, Burger M, et al. European Association of Urology Guidelines on Upper Urinary Tract Urothelial Carcinoma: 2020 Update. Eur. Urol.2020.

7. R,Yakoubi, $P$, Colin, $P, C o l i n, e t$ al. Radical nephroureterectomy versus endoscopic procedures for the treatment of localised upper tract urothelial carcinoma: A meta-analysis and a systematic review of current evidence from comparative studies - ScienceDirect. Eur J Surg Oncol. 2014, 40(12):16291634. 
8. Goel M C, Mahendra V, Roberts J G. Percutaneous Management Of Renal Pelvic Urothelial Tumors: Long-Term Followup. J. Urol.2003, 169(3):925-9; discussion 929-30.

9. Fang $D$, Gong $Y$ Q, Singla N, et al. The significance of the initial symptom in Chinese patients with upper tract urothelial carcinoma: Regular health examination is still underutilized. Kaohsiung J. Med. Sci. 2018:S1607551X17305879.

10. Chertow $\mathrm{G} \mathrm{M}$, Johansen $\mathrm{K} \mathrm{L}$, Lew $\mathrm{N}$, et al. Vintage, nutritional status, and survival in hemodialysis patients. Kidney Int. 2000, 57(3):1176-1181.

11. Gakis G, Schubert T, Alemozaffar M, et al. Update of the ICUD-SIU consultation on upper tract urothelial carcinoma 2016: treatment of low-risk upper tract urothelial carcinoma. World J Urol. 2016, 35(3):355-365.

12. Jacobi C A, Bonjer H J, Puttick M I, et al. Oncologic implications of laparoscopic and open surgery. Surg. Endosc. 2002, 16(3):441-445.

13. Wittich P, Steyerberg E W, Simons S, et al. Intraperitoneal tumor growth is influenced by pressure of carbon dioxide pneumoperitoneum. Surg. Endosc. 2000, 14(9):817-819.

14. Wittich $P$, Marquet $R L$, Kazemier $G$, et al. Port-site metastases after $C O(2)$ laparoscopy. Is aerosolization of tumor cells a pivotal factor? Surg. Endosc 2000, 14(2):189-92.

15. Micali S, Celia A, Bove P, et al. Tumor seeding in urological laparoscopy: an international survey. J. Urol. 2004, 171(6):2151-2154.

16. Yinghao Sun. Wu Jieping Urology. 2019: 792-793.

17. Ji Xu, Xiao Yu, Ying Ma et al.Dose effect relationship of the stilled water with the elimination of the exfoliated cancer cells in the peritoneal cavity during hepatocellular carcinoma resection.Chin. Med. J.2016, 96(046):3702-3705.

18. Seisen T, Colin P, M Rouprêt. Risk-adapted strategy for the kidney-sparing management of upper tract tumours. Nat Rev Urol.2015, 12(3):155.

\section{Figures}




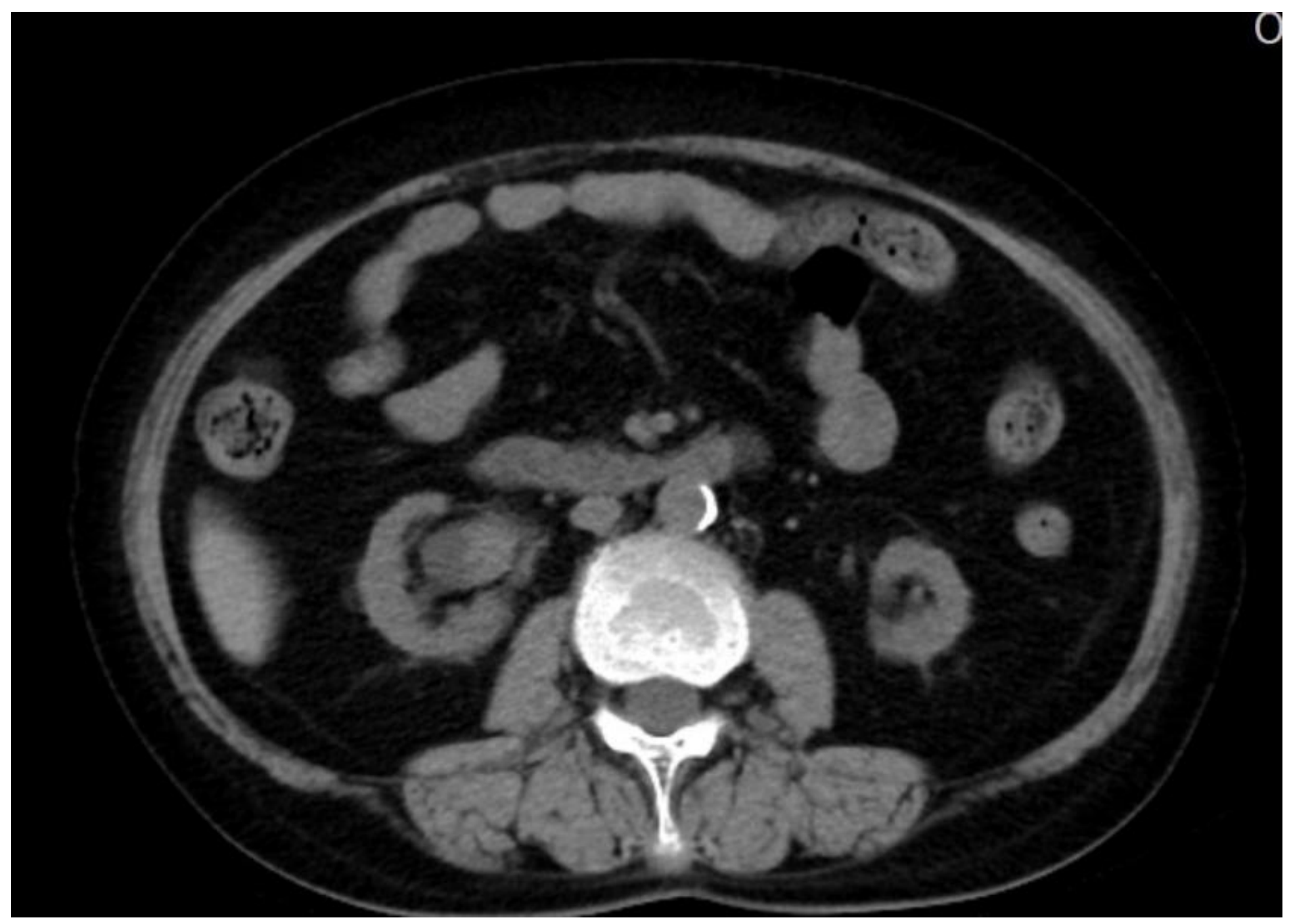

Figure 1

There is a space occupying lesion at the junction of the right renal pelvis and ureter. 


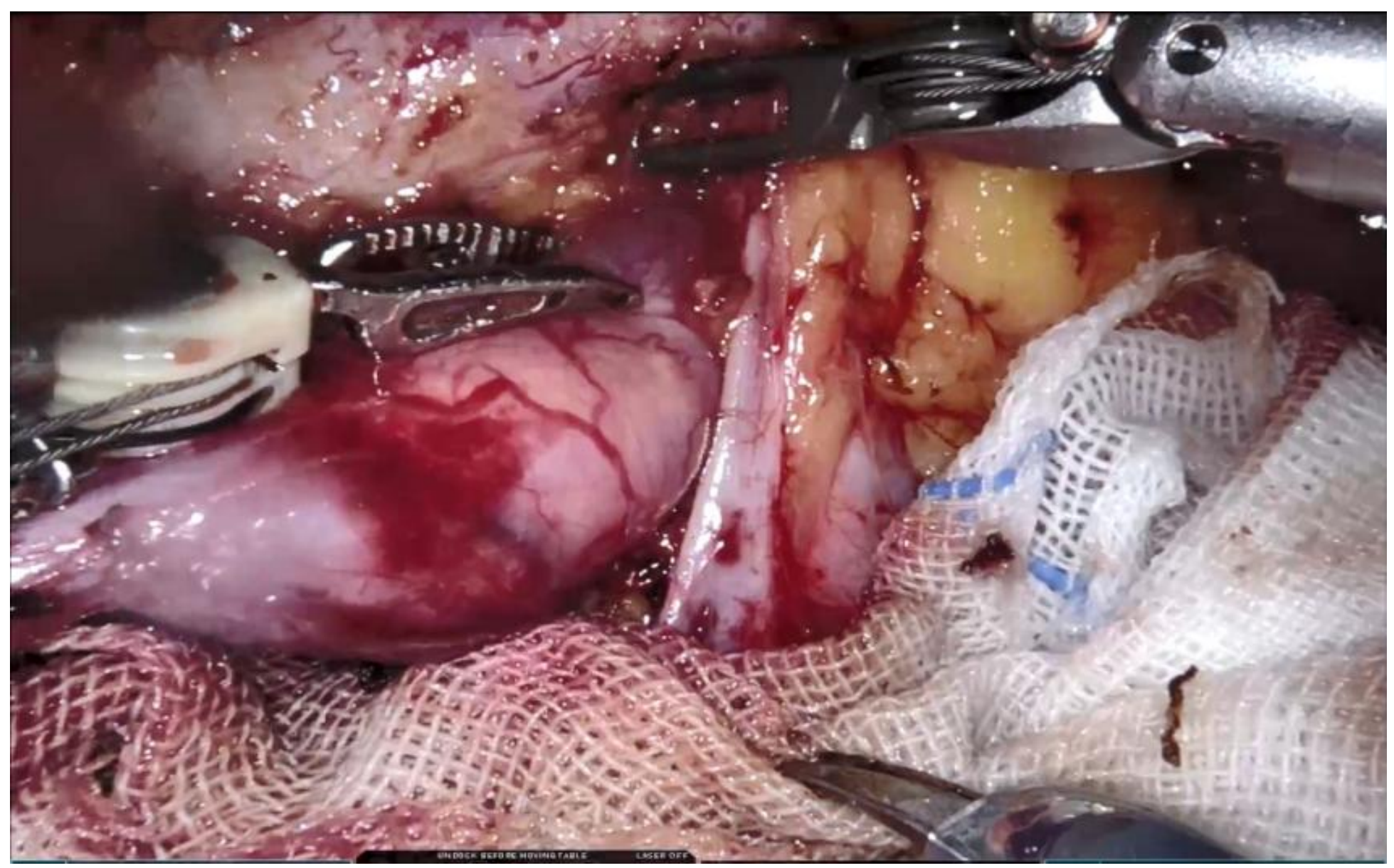

Figure 2

Dry gauze is placed around the renal pelvis to prevent spillage of urine contaminating surrounding tissue. 


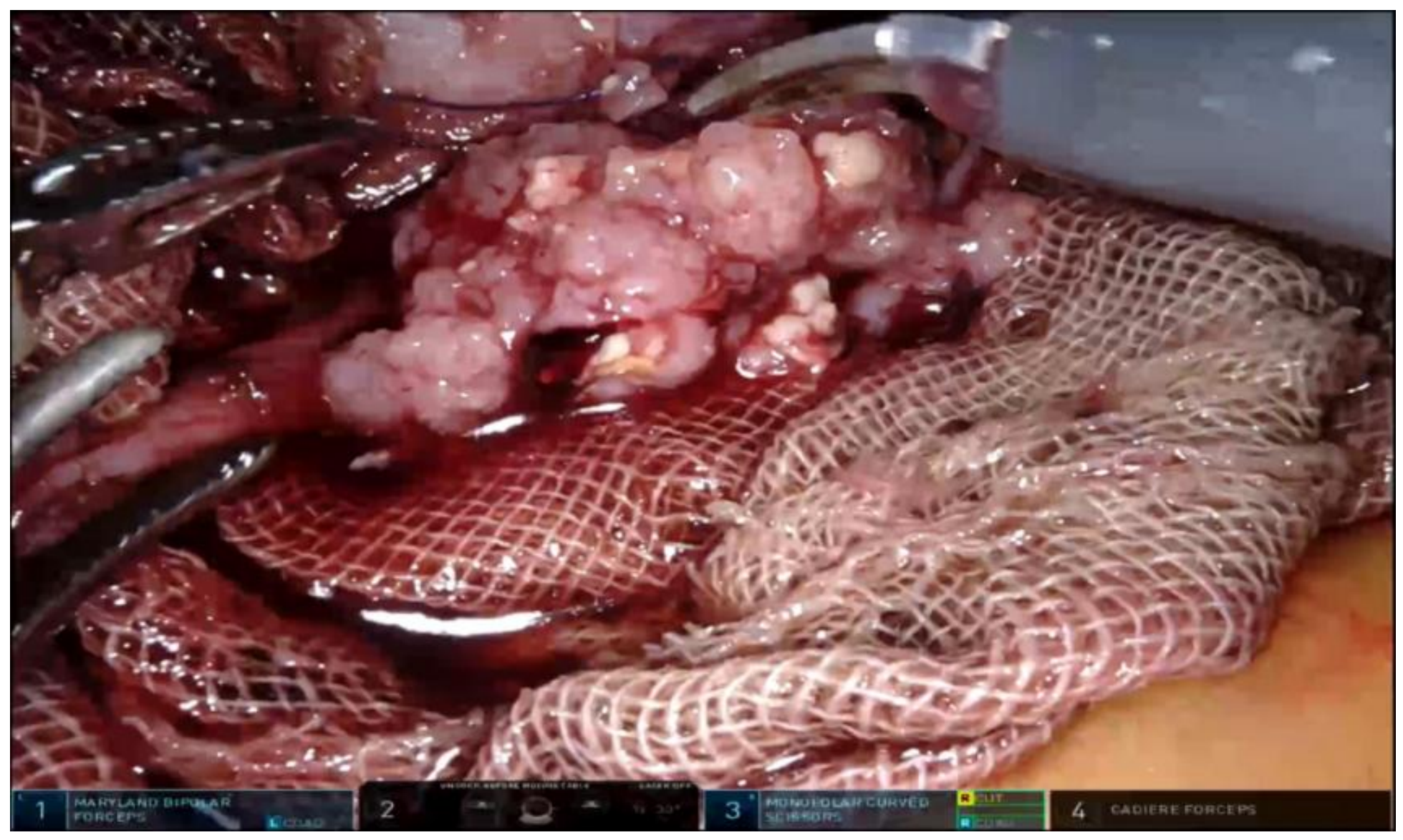

Figure 3

A vegetable-shaped tumor with a diameter of about $3 \mathrm{~cm}$ and brittle texture can be seen.

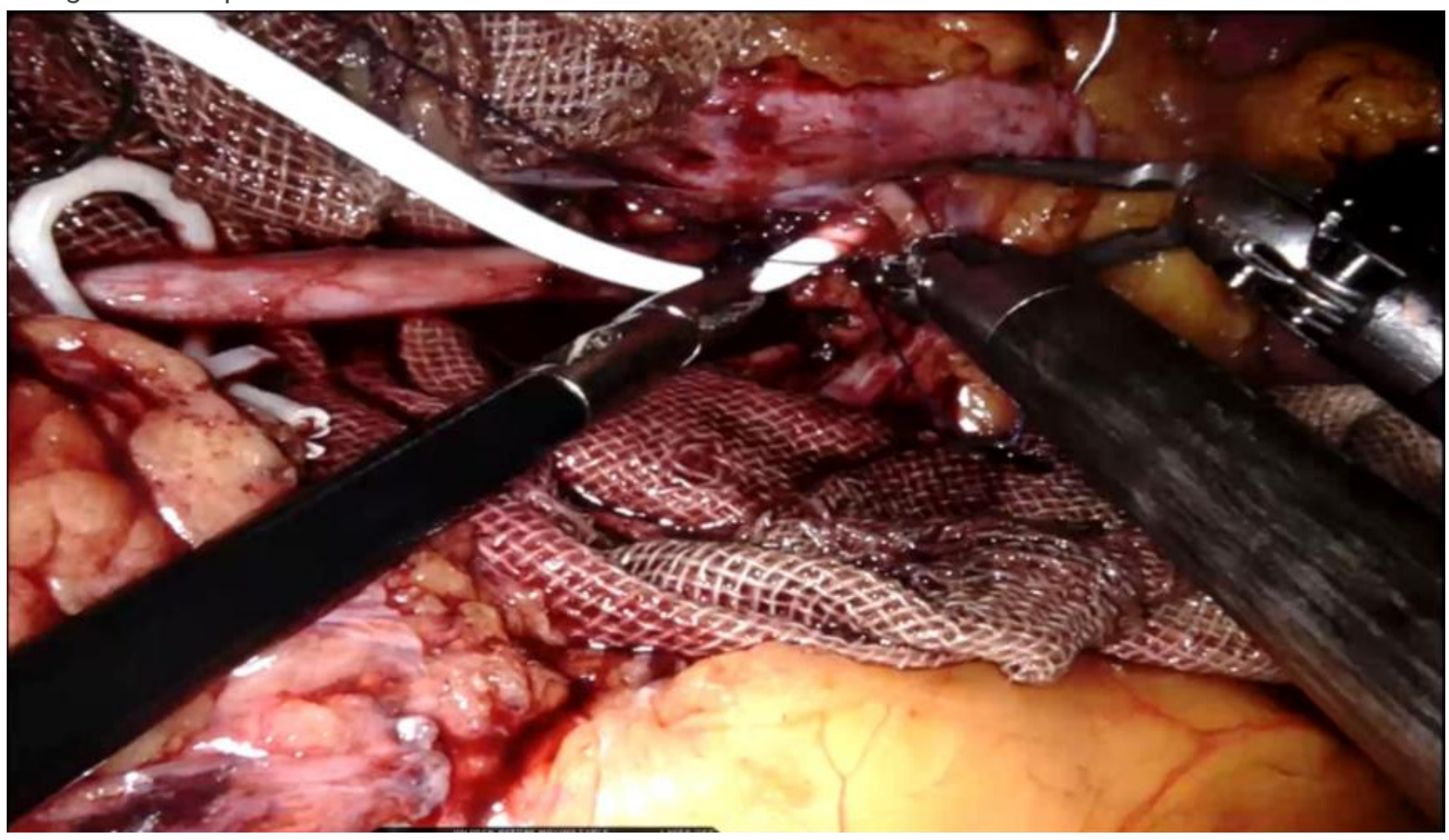


Figure 4

The single-j tube and double- $J$ tube were placed and the renal pelvis was sutured. 